\title{
INDECOMPOSABLE 1-FACTORIZATIONS OF THE COMPLETE MULTIGRAPH
}

\author{
CHARLES J. COLBOURN, MARLENE J. COLBOURN \\ and ALEXANDER ROSA
}

(Received 23 April 1984)

Communicated by W. D. Wallis

\begin{abstract}
The existence of 1-factorizations of the complete multigraph $\lambda K_{n}$ which cannot be decomposed into 1 -factorizations with smaller $\lambda$ is studied.

1980 Mathematics subject classification (Amer. Math. Soc.): 05 B 15
\end{abstract}

\section{Introduction}

Any 1-factorization of the complete graph $K_{2 n}$ provides a schedule for the $2 n-1$ rounds of a simple round robin tournament for $2 n$ teams, with each team meeting each other team exactly once. If each team is to meet each other team exactly $\lambda$ times, one schedule for such a multiple round robin tournament is obtained by combining any $\lambda$ schedules (whether identical or not) for a single round robin tournament. In graph-theoretic terms, combining any $\lambda 1$-factorizations of $K_{2 n}$ yields a 1-factorization of $\lambda K_{2 n}$.

One might ask the converse question: given a 1-factorization of $\lambda K_{2 n}, \lambda>1$, is it the union of $\lambda 1$-factorizations of $K_{2 n}$ ? It is readily seen that the answer can be "no"; it suffices to take the 15 distinct 1 -factors of $K_{6}$, remove the 51 -factors of the unique 1-factorization, and observe that the remaining 101 -factors cannot be partitioned into two 1-factorizations of $K_{6}$. A more general question would be as

(C) 1985 Australian Mathematical Society $0263-6115 / 85 \$ A 2.00+0.00$ 
follows: given a 1-factorization of $\lambda K_{2 n}, \lambda>1$, can it be written as a union of 1-factorizations of $\lambda^{\prime} K_{2 n}$ and $\lambda^{\prime \prime} K_{2 n}$ for some $\lambda^{\prime}, \lambda^{\prime \prime}<\lambda$, for which $\lambda^{\prime}+\lambda^{\prime \prime}=\lambda$ ? If a 1-factorization cannot be written in this way, we call it indecomposable. We examine the existence of indecomposable 1-factorizations of $K_{2 n}$ in this paper, and show that there exist indecomposable 1-factorizations of $\lambda K_{2 n}$ for arbitrarily high values of $\lambda$. We also settle existence of indecomposable 1-factorizations for $2 \leqslant \lambda \leqslant 6$, leaving a few small open cases.

\section{Main results}

A 1-factorization of the complete multigraph $\lambda K_{2 n}$ is a pair $(V, F)$ where $V$ is the vertex set of $K_{2 n}$, and $F$ is a collection of $\lambda(2 n-1) 1$-factors. A comprehensive survey of research on 1-factorizations of complete graphs is given in [3]. If no two members of $F$ are identical as 1-factors (i.e., no 1-factors are "repeated"), the 1 -factorization is said to be simple. We denote a 1-factorization of $\lambda K_{2 n}$ by $O F(2 n, \lambda)$; when it is indecomposable, we denote it by $\operatorname{IOF}(2 n, \lambda)$.

In what follows we need an auxiliary result on the existence of 1-factorizations of certain graphs. For $x \in Z_{n}$, define $|x|$ as $x$ if $0 \leqslant x \leqslant\lfloor n / 2\rfloor$, and $-x$ if $\lfloor n / 2\rfloor<x<n$. For $n \geqslant 2$ and $L \subseteq\{1,2, \ldots,\lfloor n / 2\rfloor\}$, let $G(n, L)$ be the regular graph with vertex set $Z_{n}$ and edge set $E$ given by $\{x, y\} \in E$ if and only if $|x-y| \in L$.

LeMma 1. Let $n$ be an even positive integer, and let $\varnothing \neq L \subseteq\{1,2, \ldots, n / 2\}$. Then $G(n, L)$ has a 1-factorization if and only if $n / \operatorname{gcd}(j, n)$ is even for at least one $j \in L$.

Proof. See $[2,4]$.

Our first result shows that there are indecomposable 1 -factorizations with arbitrarily high index $\lambda$.

TheOReM 2. There exists a simple $\operatorname{IOF}(2 n, n-1)$ whenever $2 n-1$ is a prime.

Proof. Let $V=Z_{2 n-1} \cup\{\infty\}$, and let $\theta$ be a generator of $Z_{2 n-1}$. Let $F$ be the 1 -factor $\{\{2 i-1,2 i\} \mid 1 \leqslant i<n\} \cup\{\{0, \infty\}\}$. Let $F_{i}=\theta^{i} F, 0 \leqslant i \leqslant n-2$, and let $F F=\left\{F_{i} \mid 0 \leqslant i \leqslant n-2\right\} \bmod 2 n-1$. Then $(V, F F)$ is an $O F(2 n, n-1)$, which by construction is simple. Let us show that it is also indecomposable. Assume that there exists an $O F(2 n, \lambda)\left(V, F^{\prime}\right)$ with $F^{\prime} \subseteq F F, \lambda<n-1$. Consider all of the pairs $\{x, y\}, x, y \in Z_{2 n-1}$ having $|x-y|=1$. There are $2 n-1$ 
such pairs, each of which is contained in exactly $\lambda$ factors of $F^{\prime}$. On the other hand, $F$ ' contains $m 1$-factors for some $m<2 n-1$ whose edges $\{x, y\}$ are such that $|x-y|=1$, and each of these 1-factors contains $2 n-2$ such edges. Thus we have $\lambda(2 n-1)=m(2 n-2)$, which is a contradiction.

Any nonempty set of edges of a 1-factor $F$ is a subfactor of $F$. An $\operatorname{OF}(2 n, \lambda)$ $(V, F)$ is said to be a sub-OF of an $O F(2 s, \lambda)(W, G)$ if $V \subseteq W$, and for each $f \in F$ there is a $g \in G$ such that $f$ is a subfactor of $g$. We also say that $(V, F)$ is embedded in $(W, G)$.

THEOREM 3. Any $O F(2 n, \lambda)$ can be embedded in a simple $O F(2 s, \lambda)$ for $s \geqslant 2 n$ provided $\lambda \leqslant 2 n-1$.

Proof. Let $(V, F)$ be an $O F(2 n, \lambda)$ with $V=\left\{v_{1}, v_{2}, \ldots, v_{2 n}\right\}$ and $F=$ $\left\{F_{i, j} \mid 1 \leqslant i \leqslant 2 n-1,1 \leqslant j \leqslant \lambda\right\}$. Note that $(V, F)$ is not required to be simple. However, we may assume without loss of generality that if $(V, F)$ contains repeated 1 -factors whenever $F_{i, j}$ and $F_{k, l}$ are identical as 1-factors, then $i \neq k$.

Let $w=s-n$, and consider the complete graph $K_{2 w}$ with vertex set $Z_{2 w}$ (we assume here that $\left.V \cap Z_{2 w}=\varnothing\right)$. The graph $G(2 w,\{w-n+1, w-n+$ $2, \ldots, w\})$ is regular of degree $2 n-1$, and by Lemma 1 has a 1 -factorization. Let $H_{i}, 1 \leqslant i \leqslant 2 n-1$ be the 1-factors of such a 1-factorization. We construct a set $K$ of 1 -factors on the $2 s$ vertices $V \cup Z_{2 w}$, taking $K=\left\{K_{i, j}=F_{i, j} \cup H_{i} \mid 1 \leqslant i \leqslant\right.$ $2 n-1,1 \leqslant j \leqslant \lambda\}$. $K$ is a set of $\lambda(2 n-1)$ distinct 1 -factors.

The remaining 1-factors involve edges between $V$ and $Z_{2 w}$, and are constructed as follows. Let $A=\left\{A_{r} \mid 1 \leqslant r \leqslant w-n\right\}$ be a set of $w-n$ disjoint pairs: $A_{r}=$ $\left\{a_{r}, b_{r}\right\}, a_{r}, b_{r} \in Z_{2 w},\left|a_{r}-b_{r}\right|=r, A_{r} \cap A_{q}=\varnothing$ for $r \neq q$. Such a set $A$ always exists and is easy to construct by taking a Skolem or hooked Skolem $(w-1)$ sequence and omitting from it the $n-1$ pairs with largest differences $w-n+$ $1, \ldots, w-1$.

Let $Y=\left\{y_{1}, \ldots, y_{2 n}\right\}=Z_{2 w}-\bigcup_{r=1}^{w-n} A_{r}$. Define, for $i \in Z_{2 w}, M_{i}=\left\{\left\{v_{t}, y_{t}+\right.\right.$ i\} $\mid 1 \leqslant t \leqslant 2 n\} \cup\left\{\left\{a_{r}+i, b_{r}+i\right\} \mid 1 \leqslant r \leqslant w-n\right\}$. Clearly $M_{i}$ is a 1 -factor of $K_{2 s}$ on $V \cup Z_{2 w}$. Now let $C$ be any $\lambda \times 2 n$ Latin rectangle, and let $b_{j}$ be the permutation given by the $j$ th row of $C$. Let $M_{i, j}=\left\{\left\{v_{t}, y_{t b_{j}}+i\right\} \mid 1 \leqslant t \leqslant 2 n\right\} \cup$ $\left\{\left\{a_{r}+i, b_{r}+i\right\} \mid 1 \leqslant r \leqslant w-n\right\}$. It is straightforward to verify that $M=$ $\left\{M_{i, j} \mid i \in Z_{2 w}, 1 \leqslant j \leqslant \lambda\right\}$ is a set of $2 w \lambda$ distinct 1 -factors, and further that $\left(V \cup Z_{2 w}, K \cup M\right)$ is a simple $O F(2 s, \lambda)$ containing the (not necessarily simple) $O F(2 n, \lambda)(V, F)$.

COROLlaRy 4. If there exists an $\operatorname{IOF}(2 n, \lambda)$ with $\lambda \leqslant 2 n-1$, there exists a simple IOF $(2 s, \lambda)$ for $s \geqslant 2 n$. 
Before proceeding further, we observe that any $O F(4, \lambda), \lambda>1$, is trivially decomposable. Thus if an $\operatorname{IOF}(2 n, \lambda)$ exists for $\lambda>1$, then $n \geqslant 3$.

THEOREM 5. A simple $\operatorname{IOF}(2 n, 2)$ exists if and only if $n \geqslant 3$.

Proof. An $\operatorname{IOF}(6,2)$ exists by Theorem 2 (and also by the remarks in the introduction). Theorem 3 then gives a simple $\operatorname{IOF}(2 n, 2)$ for all $n \geqslant 6$. It remains only to exhibit solutions for $n=4$ and 5 . One simple $\operatorname{IOF}(8,2)$ has $V=Z_{7} \cup$ $\{\infty\}$, and $F=F^{\prime} \cup F^{\prime \prime}$ where $F^{\prime}=\{\{0, \infty\},\{1,6\},\{2,3\},\{4,5\} \bmod 7\}$ and $F^{\prime \prime}=\{\{0, \infty\},\{1,5\},\{2,4\},\{3,6\} \bmod 7\}$.

An $\operatorname{IOF}(10,2)$ is developed similarly with $F^{\prime}=\{\{0, \infty\},\{1,4\},\{2,6\},\{3,7\}$, $\{5,8\} \bmod 9\}$ and $F^{\prime \prime}=\{\{0, \infty\},\{1,3\},\{2,4\},\{5,6\},\{7,8\} \bmod 9\}$.

THEOREM 6. A simple $\operatorname{IOF}(2 n, 3)$ exists if and only if $n \geq 4$.

Proof. An exhaustive search easily verifies that there is no $\operatorname{IOF}(6,3)$, whether simple or not. Theorem 2 yields a simple $\operatorname{IOF}(8,3)$, and then Theorem 3 gives a simple $\operatorname{IOF}(2 n, 3)$ for every $n \geqslant 8$. It remains only to give simple $\operatorname{IOF}(2 n, 3)$ for $n=5,6$, and 7 ; these are given in the appendix.

THEOREM 7. A simple $\operatorname{IOF}(2 n, 4)$ exists if and only if $n \geqslant 4$.

Proof. Necessity is obvious. For sufficiency, Theorem 3 together with a simple $\operatorname{IOF}(2 n, 4)$ for $n=4,5,6$, and 7 is enough; these $I O F$ s are given in the appendix.

THEOREM 8. A simple IOF $(2 n, 5)$ exists for $n=5,6,7$ and all $n \geqslant 10$.

Proof. A simple $\operatorname{IOF}(12,5)$ exists by Theorem 2, and a simple $\operatorname{IOF}(10,5)$ and $\operatorname{IOF}(14,5)$ are given in the appendix; Theorem 3 then gives simple $\operatorname{IOF}(2 n, 5)$ for all $n \geqslant 10$.

THEOREM 9. A simple IOF $(2 n, 6)$ exists for all $n \geqslant 6$.

Proof. A simple $\operatorname{IOF}(14,6)$ exists by Theorem 2 , and a simple $\operatorname{IOF}(12,6)$ is given in the appendix. Theorem 3 , together with a nonsimple $\operatorname{IOF}(8,6)$ given in the appendix, give simple $\operatorname{IOF}(2 n, 6)$ for all $n \geqslant 8$.

Of course, the application of the techniques developed does not merely apply to small values of $\lambda$; for example, we have 
TheOREM 10. (i) $A$ simple $\operatorname{IOF}(2 n, \lambda)$ for $\lambda=8$ or 9 exists for $n=6,7$ and all $n \geqslant 12$.

(ii) $A$ simple $\operatorname{IOF}(2 n, 10)$ exists for $n=7$ and all $n \geqslant 14$.

(iii) $A$ simple $\operatorname{IOF}(2 n, 12)$ exists for all $n \geqslant 16$.

Proof. Simple $\operatorname{IOF}(12,8), \operatorname{IOF}(14,8), \operatorname{IOF}(12,9), \operatorname{IOF}(14,9)$ and $\operatorname{IOF}(14,10)$ and a nonsimple $\operatorname{IOF}(16,12)$ are given in the appendix. The rest follows from Theorem 3.

\section{Conclusions and open problems}

There are exactly three nonisomorphic $O F(6,2)$ 's of which exactly one is indecomposable [5]. There exists no indecomposable $\operatorname{IOF}(6,3)$, whether simple or not. This can be determined by exhaustive search. Virtually nothing else is known about the enumeration of $O F(2 n, \lambda)$ 's for $\lambda>1$.

One might ask what is the maximum $\lambda=\lambda(2 n)$ such that there exists a simple $\operatorname{IOF}(2 n, \lambda)$. Taking all distinct 1-factors of $K_{2 n}$ obviously produces a simple $O F(2 n,(2 n-3) ! !)$, where $n ! !$ is the product of all odd numbers up to $n$. Thus $\lambda(2 n) \leqslant(2 n-3) ! !-1$. One has $\lambda(6)=2$, but nothing else seems to be known about $\lambda(2 n)$.

Let us mention one other (undoubtedly difficult) problem concerning 1-factorizations of $\lambda K_{2 n}$. Suppose $P=\left(p_{1}, p_{2}, \ldots, p_{k}\right)$ is a partition of the number $(2 n-3)$ !!. Is it possible to partition the 1-factors of $K_{2 n}$ on $V$ into subsets $F_{1}, \ldots, F_{k}$ such that each $\left(V, F_{i}\right)$ is an $\operatorname{IOF}\left(2 n, p_{i}\right)$ ? Let us call $P$ admissible if the answer is yes. It is easily seen that $(1,2)$ is the only admissible partition for $n=3$. Cameron [1] has shown that for $n=4$, the partition (1*15) is admissible but it follows from Theorems 5-7 that many other partitions are admissible for $n=4$.

\section{Acknowledgments}

This research was supported by the Natural Sciences and Engineering Research Council of Canada under grants numbered A5047 (CJC), A0579 (CJC), A5483 (MJC), A0764 (MJC) and A7268 (AR).

\section{Appendix}

We list here the "base" 1-factors for the $\operatorname{IOF}(2 n, \lambda)$ 's referred to in Section 2. These were produced using a straightforward backtracking algorithm by computer. The vertex set is always taken to be $Z_{2 n-1} \cup\{\infty\}$. 
Simple $\operatorname{IOF}(10,3)$

$$
\begin{array}{lllll}
\{0, \infty\} & \{1,8\} & \{2,3\} & \{4,5\} & \{6,7\} \\
\{0, \infty\} & \{1,3\} & \{2,7\} & \{4,6\} & \{5,8\} \\
\{0, \infty\} & \{1,6\} & \{2,5\} & \{3,8\} & \{4,7\}
\end{array}
$$

Simple $\operatorname{IOF}(12,3)$

$$
\begin{array}{cccccc}
\{0, \infty\} & \{1,7\} & \{2,3\} & \{4,5\} & \{6,10\} & \{8,9\} \\
\{0, \infty\} & \{1,7\} & \{2,9\} & \{3,5\} & \{4,6\} & \{8,10\} \\
\{0, \infty\} & \{1,4\} & \{2,7\} & \{3,10\} & \{5,8\} & \{6,9\}
\end{array}
$$

Simple $\operatorname{IOF}(14,3)$

$$
\begin{array}{ccccccc}
\{0, \infty\} & \{1,12\} & \{2,3\} & \{4,5\} & \{6,7\} & \{8,10\} & \{9,11\} \\
\{0, \infty\} & \{1,10\} & \{2,11\} & \{3,12\} & \{4,7\} & \{5,8\} & \{6,9\} \\
\{0, \infty\} & \{1,7\} & \{2,10\} & \{3,8\} & \{4,9\} & \{5,11\} & \{6,12\}
\end{array}
$$

Simple $\operatorname{IOF}(8,4)$

$$
\begin{array}{llll}
\{0, \infty\} & \{1,2\} & \{3,6\} & \{4,5\} \\
\{0, \infty\} & \{1,4\} & \{2,3\} & \{5,6\} \\
\{0, \infty\} & \{1,6\} & \{2,4\} & \{3,5\} \\
\{0, \infty\} & \{1,5\} & \{2,4\} & \{3,6\}
\end{array}
$$

Simple $\operatorname{IOF}(10,4)$

$$
\begin{array}{lllll}
\{0, \infty\} & \{1,2\} & \{3,4\} & \{5,6\} & \{7,8\} \\
\{0, \infty\} & \{1,4\} & \{2,7\} & \{3,5\} & \{6,8\} \\
\{0, \infty\} & \{1,7\} & \{2,6\} & \{3,5\} & \{4,8\} \\
\{0, \infty\} & \{1,3\} & \{2,6\} & \{4,7\} & \{5,8\}
\end{array}
$$

\section{Simple $\operatorname{IOF}(12,4)$}

$$
\begin{array}{cccccc}
\{0, \infty\} & \{1,2\} & \{3,4\} & \{5,10\} & \{6,7\} & \{8,9\} \\
\{0, \infty\} & \{1,10\} & \{2,8\} & \{3,5\} & \{4,6\} & \{7,9\} \\
\{0, \infty\} & \{1,4\} & \{2,5\} & \{3,8\} & \{6,9\} & \{7,10\} \\
\{0, \infty\} & \{1,7\} & \{2,6\} & \{3,10\} & \{4,8\} & \{5,9\}
\end{array}
$$


Simple $\operatorname{IOF}(14,4)$

$$
\begin{array}{ccccccc}
\{0, \infty\} & \{1,2\} & \{3,4\} & \{5,11\} & \{6,12\} & \{7,8\} & \{9,10\} \\
\{0, \infty\} & \{1,3\} & \{2,10\} & \{4,12\} & \{5,7\} & \{6,8\} & \{9,11\} \\
\{0, \infty\} & \{1,8\} & \{2,5\} & \{3,6\} & \{4,11\} & \{7,10\} & \{9,12\} \\
\{0, \infty\} & \{1,6\} & \{2,10\} & \{3,12\} & \{4,8\} & \{5,9\} & \{7,11\}
\end{array}
$$

Simple $\operatorname{IOF}(10,5)$

$$
\begin{array}{lllll}
\{0, \infty\} & \{1,2\} & \{3,4\} & \{5,6\} & \{7,8\} \\
\{0, \infty\} & \{1,5\} & \{2,6\} & \{3,7\} & \{4,8\} \\
\{0, \infty\} & \{1,4\} & \{2,3\} & \{5,7\} & \{6,8\} \\
\{0, \infty\} & \{1,7\} & \{2,5\} & \{3,6\} & \{4,8\} \\
\{0, \infty\} & \{1,7\} & \{2,4\} & \{3,5\} & \{6,8\}
\end{array}
$$

Simple $\operatorname{IOF}(14,5)$

$$
\begin{array}{ccccccc}
\{0, \infty\} & \{1,8\} & \{2,3\} & \{4,5\} & \{6,7\} & \{9,10\} & \{11,12\} \\
\{0, \infty\} & \{1,3\} & \{2,4\} & \{5,7\} & \{6,12\} & \{8,10\} & \{9,11\} \\
\{0, \infty\} & \{1,11\} & \{2,12\} & \{3,10\} & \{4,7\} & \{5,8\} & \{6,9\} \\
\{0, \infty\} & \{1,10\} & \{2,11\} & \{3,7\} & \{4,8\} & \{5,9\} & \{6,12\} \\
\{0, \infty\} & \{1,6\} & \{2,8\} & \{3,11\} & \{4,9\} & \{5,10\} & \{7,12\}
\end{array}
$$

Nonsimple $\operatorname{IOF}(8,6)$

$$
\begin{array}{llllc}
\{0, \infty\} & \{1,2\} & \{3,4\} & \{5,6\} & \text { twice } \\
\{0, \infty\} & \{1,4\} & \{2,5\} & \{3,6\} & \\
\{0, \infty\} & \{1,3\} & \{2,5\} & \{4,6\} & \text { three times }
\end{array}
$$

Simple $\operatorname{IOF}(12,6)$

$$
\begin{array}{llllll}
\{0, \infty\} & \{1,2\} & \{3,4\} & \{5,6\} & \{7,9\} & \{8,10\} \\
\{0, \infty\} & \{1,3\} & \{2,4\} & \{5,6\} & \{7,8\} & \{9,10\} \\
\{0, \infty\} & \{1,4\} & \{2,5\} & \{3,6\} & \{7,9\} & \{8,10\} \\
\{0, \infty\} & \{1,4\} & \{2,8\} & \{3,6\} & \{5,9\} & \{7,10\} \\
\{0, \infty\} & \{1,5\} & \{2,9\} & \{3,7\} & \{4,8\} & \{6,10\} \\
\{0, \infty\} & \{1,6\} & \{2,7\} & \{3,8\} & \{4,9\} & \{5,10\}
\end{array}
$$


Simple $\operatorname{IOF}(12,8)$

$\begin{array}{lccccc}\{0, \infty\} & \{1,2\} & \{3,4\} & \{5,10\} & \{6,7\} & \{8,9\} \\ \{0, \infty\} & \{1,2\} & \{3,8\} & \{4,5\} & \{6,7\} & \{9,10\} \\ \{0, \infty\} & \{1,5\} & \{2,7\} & \{3,9\} & \{4,8\} & \{6,10\} \\ \{0, \infty\} & \{1,6\} & \{2,7\} & \{3,10\} & \{4,8\} & \{5,9\} \\ \{0, \infty\} & \{1,9\} & \{2,7\} & \{3,6\} & \{4,10\} & \{5,8\} \\ \{0, \infty\} & \{1,10\} & \{2,4\} & \{3,5\} & \{6,8\} & \{7,9\} \\ \{0, \infty\} & \{1,9\} & \{2,10\} & \{3,6\} & \{4,7\} & \{5,8\} \\ \{0, \infty\} & \{1,10\} & \{2,6\} & \{3,5\} & \{4,8\} & \{7,9\}\end{array}$

Simple $\operatorname{IOF}(14,8)$

$\begin{array}{lcccccc}\{0, \infty\} & \{1,11\} & \{2,3\} & \{4,5\} & \{6,7\} & \{8,9\} & \{10,12\} \\ \{0, \infty\} & \{1,3\} & \{2,12\} & \{4,5\} & \{6,7\} & \{8,9\} & \{10,11\} \\ \{0, \infty\} & \{1,4\} & \{2,6\} & \{3,7\} & \{5,8\} & \{9,11\} & \{10,12\} \\ \{0, \infty\} & \{1,3\} & \{2,4\} & \{5,8\} & \{6,10\} & \{7,11\} & \{9,12\} \\ \{0, \infty\} & \{1,11\} & \{2,12\} & \{3,5\} & \{4,8\} & \{6,10\} & \{7,9\} \\ \{0, \infty\} & \{1,5\} & \{2,9\} & \{3,8\} & \{4,11\} & \{6,10\} & \{7,12\} \\ \{0, \infty\} & \{1,9\} & \{2,7\} & \{3,8\} & \{4,12\} & \{5,10\} & \{6,11\} \\ \{0, \infty\} & \{1,7\} & \{2,8\} & \{3,9\} & \{4,10\} & \{5,11\} & \{6,12\}\end{array}$

Simple $\operatorname{IOF}(12,9)$

$\begin{array}{lccccc}\{0, \infty\} & \{1,4\} & \{2,10\} & \{3,5\} & \{6,8\} & \{7,9\} \\ \{0, \infty\} & \{1,9\} & \{2,4\} & \{3,5\} & \{6,8\} & \{7,10\} \\ \{0, \infty\} & \{1,2\} & \{3,6\} & \{4,7\} & \{5,8\} & \{9,10\} \\ \{0, \infty\} & \{1,10\} & \{2,4\} & \{3,6\} & \{5,8\} & \{7,9\} \\ \{0, \infty\} & \{1,2\} & \{3,4\} & \{5,6\} & \{7,8\} & \{9,10\} \\ \{0, \infty\} & \{1,6\} & \{2,3\} & \{4,9\} & \{5,10\} & \{7,8\} \\ \{0, \infty\} & \{1,6\} & \{2,7\} & \{3,8\} & \{4,9\} & \{5,10\} \\ \{0, \infty\} & \{1,5\} & \{2,9\} & \{3,7\} & \{4,8\} & \{6,10\} \\ \{0, \infty\} & \{1,7\} & \{2,6\} & \{3,10\} & \{4,8\} & \{6,10\}\end{array}$


Simple $\operatorname{IOF}(14,9)$

$\begin{array}{lcccccc}\{0, \infty\} & \{1,4\} & \{2,3\} & \{5,7\} & \{6,8\} & \{9,10\} & \{11,12\} \\ \{0, \infty\} & \{1,2\} & \{3,6\} & \{4,5\} & \{7,9\} & \{8,10\} & \{11,12\} \\ \{0, \infty\} & \{1,2\} & \{3,4\} & \{5,8\} & \{6,7\} & \{9,11\} & \{10,12\} \\ \{0, \infty\} & \{1,11\} & \{2,4\} & \{3,6\} & \{5,8\} & \{7,9\} & \{10,12\} \\ \{0, \infty\} & \{1,9\} & \{2,10\} & \{3,6\} & \{4,8\} & \{5,12\} & \{7,11\} \\ \{0, \infty\} & \{1,9\} & \{2,11\} & \{3,7\} & \{4,10\} & \{5,8\} & \{6,12\} \\ \{0, \infty\} & \{1,7\} & \{2,6\} & \{3,8\} & \{4,11\} & \{5,10\} & \{9,12\} \\ \{0, \infty\} & \{1,6\} & \{2,10\} & \{3,9\} & \{4,8\} & \{5,12\} & \{7,11\} \\ \{0, \infty\} & \{1,8\} & \{2,7\} & \{3,11\} & \{4,10\} & \{5,9\} & \{7,12\}\end{array}$

Simple $\operatorname{IOF}(14,10)$

$\begin{array}{lcccccc}\{0, \infty\} & \{1,12\} & \{2,3\} & \{4,8\} & \{5,7\} & \{6,9\} & \{10,11\} \\ \{0, \infty\} & \{1,12\} & \{2,6\} & \{3,5\} & \{4,7\} & \{8,9\} & \{10,11\} \\ \{0, \infty\} & \{1,6\} & \{2,5\} & \{3,4\} & \{7,9\} & \{8,10\} & \{11,12\} \\ \{0, \infty\} & \{1,2\} & \{3,8\} & \{4,7\} & \{5,6\} & \{9,11\} & \{10,12\} \\ \{0, \infty\} & \{1,11\} & \{2,4\} & \{3,5\} & \{6,12\} & \{7,8\} & \{9,10\} \\ \{0, \infty\} & \{1,11\} & \{2,12\} & \{3,10\} & \{4,7\} & \{5,8\} & \{6,9\} \\ \{0, \infty\} & \{1,10\} & \{2,6\} & \{3,12\} & \{4,8\} & \{5,9\} & \{7,11\} \\ \{0, \infty\} & \{1,9\} & \{2,7\} & \{3,8\} & \{4,12\} & \{5,10\} & \{6,11\} \\ \{0, \infty\} & \{1,7\} & \{2,8\} & \{3,9\} & \{4,10\} & \{5,11\} & \{6,12\} \\ \{0, \infty\} & \{1,8\} & \{2,11\} & \{3,7\} & \{4,9\} & \{5,10\} & \{6,12\}\end{array}$

Nonsimple IOF $(16,12)$

$\begin{array}{lcccccccl}\{0, \infty\} & \{1,14\} & \{2,3\} & \{4,5\} & \{6,7\} & \{8,9\} & \{10,11\} & \{12,13\} & \text { twice } \\ \{0, \infty\} & \{1,4\} & \{2,7\} & \{3,14\} & \{5,13\} & \{6,12\} & \{8,10\} & \{9,11\} & \text { five times } \\ \{0, \infty\} & \{1,6\} & \{2,14\} & \{3,7\} & \{4,10\} & \{5,13\} & \{8,11\} & \{9,12\} & \\ \{0, \infty\} & \{1,10\} & \{2,5\} & \{3,14\} & \{4,9\} & \{6,13\} & \{7,11\} & \{8,12\} & \\ \{0, \infty\} & \{1,7\} & \{2,12\} & \{3,14\} & \{4,9\} & \{5,10\} & \{6,13\} & \{8,11\} & \\ \{0, \infty\} & \{1,11\} & \{2,8\} & \{3,14\} & \{4,10\} & \{5,12\} & \{6,9\} & \{7,13\} & \\ \{0, \infty\} & \{1,9\} & \{2,10\} & \{3,7\} & \{4,14\} & \{5,13\} & \{6,12\} & \{8,11\} & \end{array}$




\section{References}

[1] P. Cameron, Parallelisms of complete designs (Cambridge University Press, Cambridge 1976).

[2] A. Hartman, "Tripling quadruple systems", Ars Combinatoria 10 (1980), 255-309.

[3] E. Mendelsohn and A. Rosa, "One-factorizations of the complete graph-a survey", J. Graph Theory, to appear.

[4] G. Stern and H. Lenz, "Steiner triple systems with given subspaces; another proof of the Doyen-Wilson theorem", Boll. Un. Mat. Ital. A (5) 17 (1980), 109-114.

[5] W. D. Wallis, “A tournament problem”, J. Austral. Math. Soc. Ser. B 24 (1983), 289-291.

Department of Computer Science

University of Waterloo

Waterloo, Ontario, N2L 3G1

Canada
Department of Mathematical Sciences

McMaster University Hamilton, Ontario, L8S 4K1

Canada 